\title{
Can COVID-19 Mortality be Predicted in the Emergency Room?
}

\author{
Fatih Güneysu' ${ }^{1}$, Necip Gokhan Guner ${ }^{1}$, Ali Fuat Erdem², Ensar Durmus ${ }^{1}$, Yesim Durgun ${ }^{1}$ and Yusuf Yurumez ${ }^{1}$ \\ ${ }^{1}$ Department of Emergency Medicine, Sakarya University Training and Research Hospital, Turkey \\ ${ }^{2}$ Department of AnesthesioIogy and Reanimation, Faculty of Medicine, Sakarya University, Turkey
}

\begin{abstract}
Objective: To investigate the association of white blood cell (WBC) counts, neutrophil, platelets, lymphocyte counts, C-reactive protein (CRP), neutrophil / lymphocyte ratio (NLR), derived NLR ratio (d-NLR), and platelet / lymphocyte ratio (PLR) at the time of first admission for mortality caused by COVID-19.

Study Design: Descritive, analytical study.

Place and Duration of Study: Department of Emergency Medicine, Sakarya University Training and Research Hospital, Turkey from March 2020 to May 2020.

Methodology: One hundred and sixty-nine patients with the diagnosis of Covid-19 were retrospectively reviewed. Patients were divided into two groups as survivors and non-survivors. Inclusion criteria were age $\geq 18$ years, RT-PCR test positivity, hospitalisation. Patients with missing data were excluded. Data regarding age, gender, WBC counts, neutrophil, platelets, and lymphocyte, CRP, NLR, d-NLR, PLR and comorbid conditions were analysed for mortality. All tests were done with a two-sided significance of $5 \%$. For each endpoint, the absolute and relative effects and their corresponding $95 \%$ confidence interval were calculated.

Results: There was a statistically significant association between neutrophil, lymphocyte, CRP, NLR, $d$-NLR and PLR values $(p=0.005, p<0.001, p<0.001, p<0.001, p<0.001$, and $p<0.001$, respectively) with mortality status of the patients. The cutoff values calculated by this analysis were 67.50 years for age, $5.12 \mathrm{~K} / \mu \mathrm{l}$ for neutrophil, $1.12 \mathrm{~K} / \mu \mathrm{l}$ for lymphocyte, $67.78 \mathrm{mg} / \mathrm{dl}$ for CRP, 3.9 for NLR, 2.55 for $\mathrm{d}-\mathrm{NLR}$, and 148.85 for PLR.

Conclusion: Altered neutrophil and lymphocyte counts, NLR, d-NLR, PLR, and CRP values can be used as early predictors of mortality in Covid-19 patients.
\end{abstract}

Key Words: Covid-19, Mortality, Emergency, NLR, d-NLR, PLR.

How to cite this article: Güneysu F, Guner NG, Erdem AF, Durmus E, Durgun Y, Yurumez Y. Can COVID-19 Mortality be Predicted in the Emergency Room?. J Coll Physicians Surg Pak 2020; 30(09):928-932 .

\section{INTRODUCTION}

In December 2019, a new virus appeared in Wuhan, China, which was called as 2019 novel coronavirus. Later, the disease was labelled as coronavirus disease-19 (COVID-19); and the virus causing it named as severe acute respiratory syndrome-2 (SARS-COV-2). ${ }^{1}$ It was revealed that the disease has a broad spectrum, ranging from clinically asymptomatic infection to death. ${ }^{2}$ The common clinical manifestations of the patients included fever, nonproductive cough, dyspnea, myalgia, fatigue, normal or decreased leukocyte counts, and radiographic evidence of pneumonia.

Correspondence to: Dr. Fatih Güneysu, Department of Emergency Medicine, Sakarya University Training and Research Hospital, Turkey

E-mail: fatihguneysu55@hotmail.com

Received: July 29, 2020; Revised: August 19, 2020;

Accepted: September 16, 2020

DOI: https://doi.org/10.29271/jcpsp.2020.09.928
Despite all the supportive interventions used in the literature, the overall mortality rate was reported to be $4.7 \%$ in COVID-19 patients and more than $10 \%$ in inpatients. ${ }^{3}$

The studies on the pathophysiology of the disease report that an inflammatory response is at the forefront. ${ }^{4}$ Therefore, recent studies on COVID-19 have focused on investigating the inflammatory response. Among the most studied inflammatory markers are peripheral white blood cell (WBC) and lymphocyte counts, neutrophil/lymphocyte ratio (NLR), C-reactive protein (CRP), procalcitonin and ferritin. ${ }^{5}$ It is seen that CRP, NLR and platelet/lymphocyte ratio (PLR) are among the most widely studied biomarkers. ${ }^{6-8}$

WBC count, NLR, derived NLR ratio (d-NLR, neutrophil count divided by WBC number minus neutrophil count) and PLR indicate systemic response in inflammation. ${ }^{7}$ Most infected patients have been reported to have lymphopenia and high neutrophil levels. ${ }^{9}$ Increased NLR is associated with increased mortality not only in infectious diseases, but also in diseases such as malignancy, acute coronary syndrome, intracerebral hemorrhage. ${ }^{10}$ 
Table I: General characteristics of patients and comparison of age, gender, laboratory parameters and comorbid diseases between survivors and non-survivors.

\begin{tabular}{|c|c|c|c|c|c|}
\hline \multirow{2}{*}{\multicolumn{2}{|c|}{ Age (years) mean $\pm \mathrm{SD}$}} & $\begin{array}{l}\text { Total patients } \\
\quad(\mathrm{n}=169)\end{array}$ & $\begin{array}{c}\text { Survivor } \\
(n=84)\end{array}$ & $\begin{array}{l}\text { Non-survivor } \\
\quad(n=85)\end{array}$ & p (\%95CI) \\
\hline & & $64.34 \pm 17.62$ & $72.02 \pm 11.39$ & $56.75 \pm 19.38$ & $<0.001(10,44-20,10)$ \\
\hline \multirow{2}{*}{ Gender n (\%) } & Female & $74(43.8)$ & $34(40.5)$ & $40(47.1)$ & \multirow{2}{*}{$0.388(0,240-0,439)$} \\
\hline & Male & $95(56.2)$ & $50(59.5)$ & $45(52.9)$ & \\
\hline $\begin{array}{l}\text { WBC (K/UL) } \\
\text { median (IQR) }\end{array}$ & Normal: 4.6-10.2 K/uL & $7.70(5.47-10.90)$ & $7.90(5.23-12.66)$ & $7.69(5.74-9.49)$ & $0.261(0,255-0,273)$ \\
\hline $\begin{array}{l}\text { Neutrophil (K/uL) } \\
\text { median (IQR) }\end{array}$ & Normal: $2.0-6.9 \mathrm{~K} / \mathrm{uL}$ & $5.21(3.34-8.48)$ & $6.45(3.61-11.43)$ & $4.80(3.05-7.25)$ & $0.005(0,004-0,007)$ \\
\hline $\begin{array}{l}\text { Platelets (K/uL) } \\
\text { median (IQR) }\end{array}$ & $\begin{array}{l}\text { Normal: } 142.0-424.0 \\
\text { K/uL }\end{array}$ & $192.00(144.50-248.50)$ & $181.00(134.00-262.75)$ & $194.00(154.50-234.50)$ & $0.660(0,655-0,673)$ \\
\hline $\begin{array}{l}\text { Lymphocytes (K/UL) } \\
\text { median (IQR) }\end{array}$ & Normal: 0.8-3.4 K/uL & $1.16(0.80-2.00)$ & $0.96(0.65-1.40)$ & $1.57(0.91-2.31)$ & $<0.001(0,000-0,000)$ \\
\hline $\begin{array}{l}\mathrm{CRP}(\mathrm{mg} / \mathrm{L}) \\
\text { median (IQR) }\end{array}$ & Normal: $0.0-5.0 \mathrm{~K} / \mathrm{uL}$ & $65.20(15.70-118.33)$ & $88.06(49.33-171.31)$ & $25.20(6.57-86.81)$ & $<0.001(0,000-0,000)$ \\
\hline \multicolumn{2}{|c|}{ NLR median (IQR) } & $4.19(2.41-8.27)$ & $6.43(3.30-14.11)$ & $2.88(1.92-5.17)$ & $<0.001(0,000-0,000)$ \\
\hline \multicolumn{2}{|c|}{ d-NLR median (IQR) } & $2.60(1.51-5.08)$ & $4.31(2.18-7.55)$ & $1.76(1.08-3.59)$ & $<0.001(0,000-0,000)$ \\
\hline \multicolumn{2}{|c|}{ PLR median (IQR) } & $150.00(96.72-273.32)$ & $176.85(125.03-308.94)$ & $113.38(83.05-218.89)$ & $<0.001(0,000-0,001)$ \\
\hline \multirow{5}{*}{$\begin{array}{l}\text { Comorbidity } \\
\mathrm{N}(\%)\end{array}$} & Diabetes mellitus & $47(27.8)$ & $29(34.5)$ & $18(21.2)$ & $0.053(0,039-0,060)$ \\
\hline & Hypertension & $83(49.1)$ & $52(61.9)$ & $31(36.5)$ & $0.001(0,001-0,001)$ \\
\hline & $\begin{array}{l}\text { Cardiovascular } \\
\text { disease }\end{array}$ & $21(12.4)$ & $13(15.5)$ & $8(9.4)$ & $0.232(0,168-0,253)$ \\
\hline & Malignancy & $11(6.5)$ & $8(9.5)$ & $3(3.5)$ & $0.114(0,102-0,132)$ \\
\hline & Chronic lung disease & $22(13)$ & $12(14.3)$ & $10(11.8)$ & $0.626(0,398-0,655)$ \\
\hline
\end{tabular}

Data arenumber (\%) or a median, unless other wise indicated. *The median values (25p-75p) were indicated because parameters did not fit the normal distribution.

Research has shown that in severe cases of COVID-19, there is an increased level of NLR. ${ }^{9}$ The d-NLR, also known as the inflammatory parameter, has a prognostic value, especially in cancer patients, similar to NLR. ${ }^{11}$ These are being investigated as useful markers to determine the prognosis of patients with viral pneumonia.

PLR has been shown to be an important parameter in predicting prognosis in COVID-19 patients, just like NLR. ${ }^{8}$ It has also been shown to be associated with mortality. ${ }^{6}$ Previous studies have confirmed that PLR is closely related to tumors, diabetes, coronary heart disease, and connective tissue diseases, and is an inflammatory indicator for the clinical diagnosis of community-acquired pneumonia. ${ }^{12}$ Valuable inflammatory markers in predicting mortality in COVID-19 pneumonia have not been fully elucidated.

The objective of this study, was to determine the association of CRP, NLR, d-NLR and PLR with the mortality caused by COVID-19 association.

\section{METHODOLOGY}

This analytical study was carried out on 169 patients who were admitted at Department of Emergency Medicine, Sakarya University Training and Research Hospital, with suspicion of COVID-19 from 13 March to 9 May 2020, and diagnosed as COVID-19 by reverse transcription-polymerase chain reaction (RT-PCR). Inclusion criteria were age $\geq 18$ years, RT-PCR test positivity, hospitalisation. Patients with missing data were excluded. The study protocol was approved by the local Ethics Committee [IRB No: 71522473/050.01.04/322].

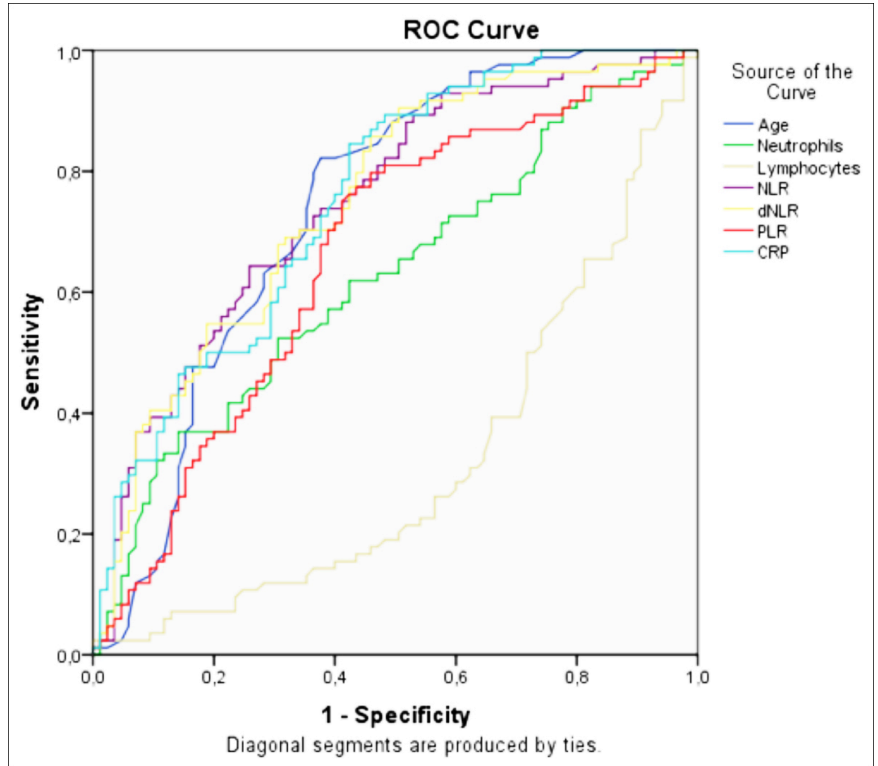

Figure 1: ROC curve was used to distinguish patients with survivor and non-survivor COVID-19.

The diagnostic process in the study was carried out according to the diagnosis and treatment guidelines of the World Health Organization and the Ministry of Health. The patients were stratified into two groups: patients who died after hospitalisation and patients who did not die after hospitalisation, and were discharged.

The data required for this study were obtained from the electronic medical records of patients in the information system of this hospital. Within the scope of the study, demographic characteristics, clinical complaints, hematological (WBC, neutrophil, platelets, lymphocytes, NLR, d-NLR, PLR) and 
serological tests (CRP), number of days of hospitalisation, throat swab samples and RT-PCR test, hospitalisation requirements, death and discharge information were recorded.

The NLR, PLR, d-NLR were calculated as NLR = ratio of neutrophil count to lymphocyte count, $d-N L R=$ ratio of neutrophil count to WBC count - neutrophil count, and PLR = ratio of platelet count to lymphocyte count.

IBM SPSS software version 21.0 was used for statistical analyses. Mean $\pm S D$ and Median (IQR) values were specified for continuous variables that did not conform to the nomal distribution. Statistical analysis of parametric and non-parametric continuous variables used independent sample t or Mann-Whitney U-test. Percentage values are given in the sharing of nominal categorical data, and statistical analyses were made by Chi-square test. Receiver operating characteristic (ROC) analysis was performed to evaluate the cutoff values of the continuous numerical variables. All tests were done with a two-sided significance of $5 \%$. For each endpoint, the absolute and relative effects and their corresponding $95 \% \mathrm{Cl}$ were calculated as recommended by Altman and colleagues.

\section{RESULTS}

General characteristics of 169 patients and the relationship regarding age, gender, laboratory parameters and comorbidity between survivors and non-survivors are shown in Table I. The mean age of the patients is $64.34 \pm 17.62$ with $56.2 \%$ males. The median values of the inflammatory markers $1.16 \mathrm{~K} / \mathrm{ul}$ (IQR: 0.80-2.00) for lymphocyte, 65.20 mg/L (IQR: 15.70-118.33) for CRP, 4.19 (IQR: 2.41-8.27) for NLR, 2.60 (IQR: 1.51-5.08) for d-NLR, and 150.00 (IQR: 96.72-273.3) for PLR, have been identified. The most common comorbidity in this study was hypertension (49.1\%); and $50.3 \%$ of patients died.

The mean age of non-survivors was $72.02 \pm 11.39$ years and those who survived was $56.75 \pm 19.38$ years. This difference is statistically significant $(p<0.001)$. There is a statistically significant difference in neutrophil, lymphocyte, CRP, NLR, dNLR and PLR values between survivors and non-survivors $(p=0.005, p<0.001, p<0.001, p<0.001, p<0.001$, and $p<0.001$, respectively).

When analysed for comorbid diseases, hypertension was statistically significantly higher in the non-survivor group $(p=0.001)$.

The ROC analysis of the inflammatory markers, that were found statistically different, was determined in Figure 1. The areas under the curve and confidence intervals are shown in Table II. Cutoff values calculated according to this analysis: 67.50 years for age, $5.12 \mathrm{~K} / \mathrm{ul}$ for neutrophil, $1.12 \mathrm{~K} / \mu \mathrm{l}$ for lymphocyte, $67.78 \mathrm{mg} / \mathrm{dl}$ for CRP, 3.9 for NLR, 2.55 for $\mathrm{d}$ NLR, and 148.85 for PLR.
The test result variables: Age, neutrophil, lymhocytes, NLR, d-NLR, PLR and CRP has at least one tie between the positive actual state group and the negative actual state group.

a. Under the non-parametric assumption.

b. Null hypothesis: True area $=0.5$.

Table II: ROC curve analysis of clinical laboratory data.

\begin{tabular}{|l|l|l|l|}
\hline Test result variables & Area & Asymptotic Sig. $^{\text {b }}$ & $\mathbf{9 5 \%} \mathbf{C l}$ \\
\hline Age & 0.739 & $<0.001$ & $0.663-0.815$ \\
\hline Neutrophil & 0.625 & 0.005 & $0.540-0.709$ \\
\hline Lymhocytes & 0.318 & $<0.001$ & $0.237-0.399$ \\
\hline NLR & 0.748 & $<0.001$ & $0.674-0.821$ \\
\hline d-NLR & 0.748 & $<0.001$ & $0.674-0.821$ \\
\hline PLR & 0.660 & $<0.001$ & $0.577-0.743$ \\
\hline CRP & 0.754 & $<0.001$ & $0.683-0.826$ \\
\hline
\end{tabular}

\section{DISCUSSION}

Since December 2019, COVID-19 has resulted in significant morbidity and mortality in more than 30 countries worldwide. This highly infectious virus has surpassed severe acute respiratory syndrome (SARS) and Middle-east respiratory syndrome in terms of mortality toll. ${ }^{13}$ The main cause of coronavirus-induced death is often considered severe respiratory distress. Studies have shown that $26 \%$ of patients are treated in intensive care, with a mortality rate of $4.3 \%-4.7 \%{ }^{2,3}$

Although COVID-19 affects any person at any age, studies have found that the frequency of death is highest in the elderly population. ${ }^{14}$ Data from China and Italy revealed that most COVID-19 deaths occurred in adults aged $\geq 60$ years. ${ }^{14}$ In this study, the mean age of the patients in the deceased group was 72 years, and that in the survivors was 58. Multivariate logistic regression analysis determined 67.5 years of age as a strong predictor for death from COVID-19related pneumonia.

In a study involving 1,099 COVID-19-confirmed patients, hypertension (23\%) and diabetes mellitus (16\%) were reported as the most common comorbid diseases. ${ }^{15} \mathrm{~A}$ study on COVID-19 mortality found that in 54 patients who died, $48 \%$ had hypertension and $17 \%$ had diabetes. ${ }^{4}$ As expected, our analysis showed that underlying hypertension and diabetes were associated with high mortality, consistent with findings of a similar study on COVID-19 pneumonia. ${ }^{16}$

It has been established that COVID-19 pneumonia is contagious, but the mechanisms by which SARS-CoV-2 causes severe illness and fatal outcomes in humans have not been established. The systemic inflammatory responses that occurs in some COVID-19 patients include increased cytokine release, blood cell component abnormalities, and clotting factor activation. ${ }^{17}$ With the activation of the inflammatory response, neutrophil production increases, while lymphocyte apoptosis accelerates. ${ }^{4}$ Lymphocytopenia has been reported to be associated with mortality in COVID-19 patients. ${ }^{5}$ In a study on COVID-19 severity, neutrophil counts 
were found to be significantly high, while lymphocyte counts were significantly low. ${ }^{18}$ In this study, high neutrophil and low lymphocyte values were found in the group of patients who died. Thus, it was established that a decrease in lymphocytes and an increase in neutrophils are important indicators for mortality. Blood tests with particular attention to these parameters should be conducted for patients admitted to the Emergency Room for COVID-19.

CRP levels indicate the severity of inflammation; and concentration levels do not change regardless of age, gender, or physical condition. ${ }^{19} \mathrm{CRP}$ activates the complement system and increases phagocytosis. ${ }^{20}$ Studies that examined CRP in COVID-19 cases reported that CRP levels obtained in the early stage of COVID-19 may predict disease severity. ${ }^{20}$ In this study, a relationship between CRP levels and mortality was found. CRP levels measured at early onset of COVID-19 may predict mortality.

Recent reports indicate that inflammatory cytokines, chemokines, and NLR are associated with disease severity and indicative of the severity of a cytokine storm. ${ }^{9} \mathrm{NLR}, \mathrm{d}$ NLR and PLR mainly indicate the level of systemic inflammation, and their relationship with virulence has been demonstrated in COVID-19 patients. ${ }^{6,8}$ High NLR has been associated with severe clinical symptoms and intensive care unit-associated admissions, recoveries, and conditions such as discharge and mechanical ventilation. ${ }^{6}$ Increased PLR has been associated with the number of days of hospitalisation and may indicate the degree of a cytokine storm. ${ }^{8}$ In this study, increased NLR, d-NLR, and PLR were found to be associated with mortality. Assessment for these parameters at the time of admission to the Emergency Room can help identify high-risk patients, and facilitate patient management.

In this study, the applicable thresholds for neutrophil, lymphocyte, CRP, NLR, d-NLR, and PLR were observed using the receiver operating characteristic curve. The optimal thresholds obtained for neutrophil, lymphocyte, CRP, NLR, PLR, and d-NLR $(5.12,1.12,67.78,3.9,148.85$, and 2.55, respectively) showed the highest probabilities of sensitivity and specificity to change among deceased and survived patients. These patients should be closely monitored by clinicians. In studies related to disease severity, the CRP cutoff value was $30.7 \mathrm{ng} / \mathrm{ml},{ }^{21}$ PLR was $126.7 \mathrm{ng} / \mathrm{ml}$, ${ }^{8}$ and NLR was $3.3 \mathrm{ng} / \mathrm{ml} .{ }^{6}$ The higher shear values of CRP, NLR, and PLR, obtained in this study and compared with other studies, indicate the bigger scope of this study in terms of the number of patients. Furthermore, cutoff values for neutrophil, lymphocyte, and d-NLR in COVID-19 pneumonia were not found in the literature; hence, this is the first such report to the best of authors' knowledge. This study has relevant contributions to the literature, given a more comprehensive scope and more conclusive results compared with other publications on COVID-19-related mortality.

\section{CONCLUSION}

The findings provide further evidence that neutrophil and lymphocyte counts, and NLR, d-NLR, PLR, and CRP values can be used as early predictors of mortality.

\section{ETHICAL APPROVAL:}

The study protocol was approved by the local Ethics Committee [IRB No:71522473/050.01.04/322].

\section{PATIENTS' CONSENT:}

Informed consents were obtained from all participants.

\section{CONFLICT OF INTEREST:}

Authors declared no conflict of interest.

\section{AUTHORS' CONTRIBUTION:}

FG: Led and conceived the project, and authored the manuscript.

NGG: Data collection, compiling, statistics and discussion.

AFE: Contributed to design articles, collected and analysed data.

ED: Contributed to collect and analyse data.

YD: Collected and analysed data, literature review and discussion.

YY: Helped perform the analyses with constructive discussions.

\section{REFERENCES}

1. Caramelo F, Ferreira N, Oliveiros B. Estimation of risk factors for COVID-19 mortality - preliminary results. MedRxiv 2020; 2020. doi.10.1101/2020.02.24.20027268.

2. Wang D, Hu B, Hu C, Zhu F, Liu X, Zhang J, et al. Clinical characteristics of 138 hospitalised patients with 2019 novel coronavirus-Infected pneumonia in Wuhan, China. JAMA 2020; 323(11):1061-9. doi: 10.1001/jama.2020.1585.

3. Zhang G, Zhang J, Wang B, Zhu X, Wang Q, Qiu S. Analysis of clinical characteristics and laboratory findings of 95 cases of 2019 novel coronavirus pneumonia in Wuhan, China: A retrospective analysis. Respir Res 2020; 21(1):74. doi: 10.1186/s12931-020-01338-8.

4. Liu Y, Du X, Chen J, Jin Y, Peng L, Wang HHX, et al. Neutrophil-to-lymphocyte ratio as an independent risk factor for mortality in hospitalized patients with COVID-19. J Infection 2020; 81(1):e6-e12. doi: 10.1016/j.jinf.2020. 04.002.

5. Zhou F, Yu T, Du R, Fan G, Liu Y, Liu Z, et al. Clinical course and risk factors for mortality of adult inpatients with COVID-19 in Wuhan, China: A retrospective cohort study. The Lancet 2020; 395(10229):1054-62. doi: 10.1016/ S0140-6736(20)30566-3.

6. Yang AP, Liu J, Tao W, Li H. The diagnostic and predictive role of NLR, d-NLR and PLR in COVID-19 patients. Int Immunopharmacol 2020; 84:106504. doi: 10.1016/ j.intimp.2020.106504

7. Ying HQ, Deng QW, He BS, Pan YQ, Wang F, Sun HL, et al. The prognostic value of preoperative NLR, d-NLR, PLR and LMR for predicting clinical outcome in surgical colorectal cancer patients. Med Oncol 2014; 31(12):305. doi: 10.1007/s12032-014-0305-0. 
8. Qu R, Ling $Y$, Zhang YH, Wei LY, Chen X, Li XM, et al. Platelet to lymphocyte ratio is associated with prognosis in patients with coronavirus disease. J Med Virol 2020; 10.1002/jmv.25767.19. doi: 10.1002/jmv.25767.

9. Qin C, Zhou L, Hu Z, Zhang S, Yang S, Tao Y, et al. Dysregulation of Immune response in Patients with coronavirus 2019 (COVID-19) in Wuhan, China. Clin Infec Dis Oxford Academic 2020; doi/10.1093/cid/ ciaa248/5803306.

10. Azab B, Zaher M, Weiserbs KF, Torbey E, Lacossiere K, Gaddam S, et al. Usefulness of neutrophil to lymphocyte ratio in predicting short- and long-term mortality after nonST-elevation myocardial infarction. Am J Cardiol 2010; 106(4):470-6. doi: 10.1016/j.amjcard.2010.03.062.

11. Proctor MJ, McMillan DC, Morrison DS, Fletcher CD, Horgan PG, Clarke SJ. A derived neutrophil to lymphocyte ratio predicts survival in patients with cancer. Br J Cancer 2012; 107(4):695-9. doi: 10.1038/bjc.2012.292.

12. Li Q, Guan X, Wu P, Wang X, Zhou L, Tong Y, et al. Early transmission dynamics in wuhan, china, of novel coronavirus-Infected pneumonia. N Engl J Med 2020; 382(13):1199-1207. doi/full/10.1056/NEJMoa2001316.

13. Shi S, Qin M, Shen B, Cai Y, Liu T, Yang F, et al. Association of cardiac Injury with mortality in hospitalised patients with COVID-19 in Wuhan, China. JAMA Cardiol 2020; 5(7):802-10. doi: 10.1001/jamacardio.2020.0950.

14. Bialek S, Boundy E, Bowen V, Chow N, Chon A, Dowling E, et al. Severe outcomes among patients with coronavirus disease 2019 (COVID-19) - United States, February 12-March 16, 2020. MMWR Morb Mortal Wkly Rep 2020; 69(12):343-6. doi: 10.15585/mmwr.mm6912e2.
15. Aggarwal S, Garcia-Telles N, Aggarwal G, Lavie C, Lippi G, Henry BM. Clinical features, laboratory characteristics, and outcomes of patients hospitalized with coronavirus disease 2019 (COVID-19): Early report from the United States. Diagnosis (Berl) 2020; 7(2):91-6. doi: 10.1515/ dx-2020- 0046.

16. Lu H, Stratton CW, Tang YW. Outbreak of pneumonia of unknown etiology in Wuhan, China: The mystery and the miracle. J Med Virolgy 2020; 92:401-2. doi.org/10. 1002/jmv.25678.

17. Fu J, Kong J, Wang W, Wu M, Yao L, Wang Z, et al. The clinical implication of dynamic neutrophil to lymphocyte ratio and D-dimer in COVID-19: A retrospective study in Suzhou China. Thromb Res 2020; 192:3-8. doi: 10.1016/ j.thromres.2020.05.006.

18. Zheng M, Gao Y, Wang G, Song G, Liu S, Sun D, et al. Functional exhaustion of antiviral lymphocytes in COVID-19 patients. Cellular \& Molecular Immunology 2020; 17(5):533-5. doi: 10.1038/s41423-020-0402-2.

19. Bilgir O, Bilgir F, Calam M, Calam OG, Yuksel A. Comparison of pre- and post-levothyroxine high-sensitivity c-reactive protein and fetuin-a levels in subclinical hypothyroidism. Clinics 2015; 70(2):97-101. doi: 10.6061/clinics/ 2015(02)05.

20. Wang L. C-reactive protein levels in the early stage of COVID-19. Médecine et Maladies Infectieuses 2020; 50(4):332-4. doi: 10.1016/j.medmal.2020.03.007.

21. Gong J, Dong H, Xia SQ, Huang YZ, Wang D, Zhao $Y$, et al. Correlation analysis between disease severity and Inflammation-related parameters in patients with COVID-19 pneumonia. Medrxiv 2020; 2020.02.25.20025643. 